\title{
Residual long-range pseudoscalar forces between unpolarised macroscopic bodies
}

\author{
J. A. Grifols \\ S. Tortosa \\ IFAE Institut de Física d'Altes Energies \\ Grup de Física Teòrica \\ Universitat Autònoma de Barcelona \\ 08193 Bellaterra, Barcelona, Catalonia, Spain
}

\begin{abstract}
In this paper we survey the effects of residual long-range forces associated to $\gamma_{5}$-spin dependent-couplings of fermions to massless bosons exerted by unpolarised bulk matter over macroscopic distances. We establish that such forces with behaviour proportional to $R^{-6}$ do indeed exist. They arise as a quantum mechanical effect due to simultaneous exchange of two quanta. We explore their presence in existing astronomical as well as laboratory data on non-newtonian components of the force between macroscopic bodies. Since no limits on their real existence could be found, we conclude that residual long-range pseudoscalar attractive and composition dependent forces between neutral unpolarised bulk matter extending over macroscopic distances are very efficiently shielded over a huge range of distances: from astronomical scales down to the micron scale.
\end{abstract}


The issue whether long-range interactions other than the already known in nature exist has been raised repeatedly and from different fronts of both experimental/observational and theoretical physics. To name just a few, the reanalysis of Eötvös experiment and all the subsequent experimental and theoretical activity boosted by a $5^{\text {th }}$ force hypothesis [1]; the dark matter problem and its feeble non gravitational interactions [2]; the hypothesised existence of very light particles (e.g. axions, majorons, etc.) to solve profound questions in particle physics, and which could mediate new interactions or the idea that baryon number (or lepton number) might be charges associated to a local gauge symmetry and thus long-range interactions should be associated to the exchange of massless vector quanta [3]. A vast domain of couplings and ranges for such forces has been explored and limits thereof have been set in terrestrial laboratories as well as astronomical/astrophysical/cosmological environments [4]. Mostly the long-range interactions for which astronomical or laboratory limits do exist are associated to vector or scalar quanta. Pseudoscalar interactions, on the other hand, cannot be directly tested in astronomy and (in general) in the laboratory using bulk matter since $\gamma_{5}$ couplings do not extend their influence over unpolarised macroscopic bodies $\rrbracket$. Of course, indirect limits in macroscopic systems on the strength of such forces do exist, involving more or less detailed knowledge on the physics of stellar evolution. The mass and coupling strengths of axions for instance have been extensively surveyed and constrained in various astrophysical or cosmological environments ranging from bounds coming from cosmic string radiation of axions to bounds derived from the cooling of white dwarfs and axion emission in Supernova collapse [5].

In the present paper we would like to explore on a quantitative basis to what extent are pseudoscalar long-range forces between unpolarised bodies screened off over macroscopic scales. To this end we shall use astronomical observations as well as laboratory experiments and we shall make no resort on stellar evolution modelling. We shall see, of course, as already mentioned above that these constraints are extremely weak and even non existing in the astronomical domain.

It is a well-known fact that spin dependent interactions do not generally extend macroscopically over large distances. Indeed, one would need polarised samples for forces to show their influence coherently over macroscopic distances. We have in mind interactions such as pseudoscalar particles coupled to nucleon sources (or, in general, coupled to fermions) or Pauli magnetic moment couplings of fermions to the electromagnetic field. In both cases, even though the range of the interaction may be infinite (for a massless pseudoscalar and obviously for the photon), macroscopic bulk matter does not feel those forces unless the samples are polarised. Actually, it is the helicity-flip nature of such interactions that prevents the exchanged quantum to extend its influence over macroscopic bodies. This fact, however, corresponds to the situation where a single quantum is exchanged "at a time", i.e. it corresponds to the ladder aproximation in a Bethe-Salpeter approach of the bound state problem. Naturally then, the question arises as to what happens if two quanta are exchanged "at a time". That is, if we go beyond the ladder approximation. Intuitively, the helicity non-flip nature is restored in this case and, consequently, one should expect coherent effects to extend over macro-

\footnotetext{
${ }^{1}$ This is true only to leading order in the interaction as we shall show below.
} 
scopic matter. In this paper we explore this possibility and find that indeed long-range macroscopic forces develop across extended bodies. Of course, as one could also foresee, these effects are extremely tiny.

We shall consider non-relativistic, non second quantised matter coupled to a pseudoscalar quantum field $\phi$. To be definite consider the interaction

$$
\mathrm{L}_{\text {int }}=\frac{g}{2 M} \chi^{\dagger} \vec{\sigma} \chi \cdot \nabla \phi(\vec{r})
$$

which is the static non-relativistic (NR) limit of $\bar{\psi}(x) \gamma_{5} \psi(x) \phi(x)$ ( $\chi$ are the Pauli spinors, $M$ is the fermion mass). Of course, if $\phi(x)$ were a scalar field then $\bar{\psi}(x) \psi(x)$ would be, in the NR-limit, just a number density of particles and hence bulk matter could act coherently as a source, whereas for the spin-flip interaction in eq (1) this is not the case. []

Now, consider two point-like matter sources a distance $\mathrm{R}$ apart at positions $\vec{R}_{1}$ and $\vec{R}_{2}, \vec{R}=\vec{R}_{1}-\vec{R}_{2}$. The interaction hamiltonian is

$$
H_{i n t}=\int d^{3} \vec{r} \sum_{i=1,2} \frac{g}{2 M} \delta\left(\vec{r}-\vec{R}_{i}\right) \vec{\sigma}^{(i)} \cdot \nabla \phi(\vec{r})
$$

where $\phi(\vec{r})=\int \frac{d^{3} \vec{k}}{(2 \pi)^{3}} \frac{1}{2 \omega_{k}}\left[a_{k} e^{-i \vec{k} \cdot \vec{r}}+a_{k}^{\dagger} e^{i \vec{k} \cdot \vec{r}}\right]$ and $\omega_{k}=\sqrt{\vec{k}^{2}+m^{2}}$ with $m$ the mass of the pseudo-scalar quantum. Eventually we shall make $m \rightarrow 0$, since we are interested in long-range effects.

In order to account for the mean effect of bulk matter we shall actually calculate $\operatorname{Tr}\left(\rho H_{\text {int }}\right)$ where $\rho$ is the density matrix operator that describes the state of the system in spin space of the two-fermion sources (we label the states that span the two-fermion space by $|i\rangle, i=1, \ldots, 4)$.

To do the calculation we shall resort to "old fashioned" perturbation theory. Since the hamiltonian in eq. (2) creates (annihilates) $\phi$ quanta from (into) the vacuum, the expectation value of $H_{\text {int }}$ between vacuum pseudo-scalar states vanishes. There are no linear effects in $g$. But second order perturbation can, in principle, connect the vacuum with the vacuum, for $H_{\text {int }}$ can create one pseudo-scalar and, on second application, annihilate it again into the vacuum.

A straightforward calculation then gives, to second order, for the interaction energy between the two sources,

$$
\Delta E_{\text {int }}^{(2)}=\frac{g^{2}}{4 M^{2}} \vec{\sigma}^{(1)} \cdot \nabla \vec{\sigma}^{(2)} \cdot \nabla \frac{e^{-m R}}{4 \pi R}
$$

where $\left(\vec{\sigma}^{(1)} \cdot\right)\left(\vec{\sigma}^{(2)} \cdot\right)$ has to be understood as an operator that acts on the $2 \times 2$ dimensional spin-space of the two-fermion system. We should point out that to reach eq.(3) we have substracted off the (infinite) self-interaction energies of each source, which furthermore are independent of $R$ and hence do not contribute to the force.

\footnotetext{
${ }^{2}$ The electromagnetic analogue of eq (1) can be derived from the Pauli interaction $\frac{\mu}{2} \bar{\psi} \sigma_{\mu \nu} \psi F^{\mu \nu}$ where $\mu$ is the magnetic moment. It reduces to $\mathrm{L}_{i n t}=\mu \chi^{\dagger} \vec{\sigma} \chi \cdot \vec{B}$ in the NR-limit.
} 
Eq.(3) is a well-known result [6] and, clearly, when we perform the spin average in the tensor product space of the two Pauli spinors that corresponds to unpolarised sources, the interaction energy vanishes since

$$
\operatorname{Tr}(\vec{\sigma} \otimes \vec{\sigma})=0
$$

(the density matrix is just $\frac{1}{4} I$ ). Thus, we expect no macroscopic effect to this order as already advertised before.

We turn now to the next order in perturbation theory, i.e. $O\left(g^{4}\right)$ since only even powers of $H_{\text {int }}$ are permitted. This corresponds to the emission and subsequent absorption of two quanta f Again we encounter in the calculation infinities associated to self-energies of the sources. Indeed, there are, to this order, the self-energies arising from the emission and absorption of one quantum by each source and emission and absorption of two quanta by either source. We simply substract off these infinities for they again do not depend on $R$ and lead to no force. But, there are also divergences corresponding to the case where one quantum is exchanged between both sources and the other one is emitted and reabsorbed by source 1 or source 2, respectively. These last terms, however, vanish when performing the statistical average over unpolarised samples.

The result, after statistical averaging is

$$
\begin{aligned}
\overrightarrow{\Delta E}_{\text {int }}^{(4)}= & -\frac{2}{(2 \pi)^{6}}\left(\frac{g}{2 M}\right)^{4} \operatorname{Tr}\left\{\left[(\vec{\sigma} \cdot \nabla \otimes \vec{\sigma} \cdot \nabla) \int \frac{d^{3} \vec{p}}{\omega_{p}^{3}} \cos (\vec{p} \cdot \vec{R})\right]\right. \\
& \left.\times\left[(\vec{\sigma} \cdot \nabla \otimes \vec{\sigma} \cdot \nabla) \int \frac{d^{3} \overrightarrow{p^{\prime}}}{\omega_{p^{\prime}}^{2}} \cos \left(\overrightarrow{p^{\prime}} \cdot \vec{R}\right)\right]\right\}
\end{aligned}
$$

which is certainly different from zero. Performing the integrals, eq.(5) can be put in the form

$$
{\overline{\Delta E_{i n t}}}_{\text {(4) }}=-\frac{1}{4 \pi^{3}}\left(\frac{g}{2 M}\right)^{4} \sum_{i, j=1}^{3}\left(\frac{\partial}{\partial R_{i}} \frac{\partial}{\partial R_{j}} K_{0}[m R]\right)\left(\frac{\partial}{\partial R_{i}} \frac{\partial}{\partial R_{j}} \frac{e^{-m R}}{R}\right)
$$

with $K_{0}$ the modified Bessel function. In the limit $m \rightarrow 0$, we obtain

$$
\overline{\Delta E}_{\text {int }}^{(4)}=-\frac{g^{4}}{16 \pi^{3} M^{4}} \frac{1}{R^{5}}
$$

for the interaction energy for two spin averaged fermion samples normalized to one fermion per sample f. The resulting force between two pieces of matter is attractive, composition dependent and very small.

We should note that the statistical averaging in eq.(5) involves the introduction of a finite renormalization of the two-fermion wavefunction. In fact, to the order we are working, the statistical weights $\omega_{i}$ in the density matrix $\rho$ are related to the lowest order density matrix elements that enter in eq.(5) through a rescaling $\omega_{i}=\omega^{(0)} Z_{i}^{-1}$, where $Z_{i}$ is the (finite) wavefunction renormalization of the perturbed states, i.e. $Z_{i}^{-1}=\langle i \mid i\rangle$.

\footnotetext{
${ }^{3}$ For work on long-range two-photon forces see [7] and references therein.

${ }^{4}$ The corresponding formula for the electromagnetic Pauli dipole interaction is $\overline{\Delta E}_{\text {int }}^{(4)}=-\frac{\mu^{4}}{\pi^{3}} \frac{1}{R^{5}}$.
} 
The new force should couple to baryons and/or leptons and hence the coupling be proportional to an arbitrary combination of baryon and lepton quantum numbers. Since the force goes as $M^{-4}$, in order to maximize the effect, we shall in what follows couple the interaction fully to the electrons in matter and thus guarantee that our bounds are the most restrictive one can get.

Already from the $R$ dependence of the force one can suspect that, on astronomical scales, the effect of pseudoscalar massless exchange will be totally negligible. Indeed, a quantity that typically supplies constraints on non-newtonian components of the force in the astronomical realm is the perihelion of Mercury. In our case, the effect would be a perihelion shift of the order $\Delta \phi \sim 2 \times 10^{-54} \alpha_{g}{ }^{2} \frac{\mathrm{rad}}{\mathrm{rev}}$ which gives no limit at all on $\alpha_{g}=\frac{g^{2}}{4 \pi}$. This result is completely general: no astronomical observation is able to put limits on pseudoscalar long-range forces.

As to laboratory experiments, at present the best laboratory limits to non-newtonian components of the force between macroscopic bodies come from the null experiments of Hoskins et al. and Chen et al. [8]. In particular, Hoskins et al. test deviations from the $\frac{1}{R^{2}}$ law in the $2-5 \mathrm{~cm}$ range. These authors place a test mass inside a long and hollow cylinder and monitor the torque on the mass as the distance between the mass and the cylinder is varied. The mass in an infinitely long cylinder would experience no gravitational force and hence one can set direct limits on non gravitational effects by performing the experiment. There are corrections, of course, due to end effects in a finite cylinder, but these are small enough to allow detection of a force deviating from the $\frac{1}{R^{2}}$ law. The actual experiment reached a sensitivity to acceleration of $2 \times 10^{-11} \frac{\mathrm{cm}}{\mathrm{s}^{2}}$ and used a $20 \mathrm{~g}$ test mass of high-purity copper and a $60 \mathrm{~cm}$ long cylinder of thickness $1 \mathrm{~cm}$ and an interior diameter of $6 \mathrm{~cm}$ made of high-purity double-vacuum-melted stainless steel. No deviation to the newtonian law was observed at the level of the sensitivity stated above. We can use these results to try to constrain our pseudoscalar force by calculating the radial acceleration towards the wall of the tube caused by our putative pseudoscalar force on the test mass suspended from the end of a torsion balance bar

$$
a=\frac{(\hbar c)^{5}}{c^{8}} \frac{5}{\pi} \frac{\alpha_{g}^{2}}{M_{e}^{6}} f_{e}^{(\text {sample })} f_{e}^{(\mathrm{cyl})} \rho^{(\mathrm{cyl})} \int_{R_{0}}^{R_{0}+d} d r \int_{0}^{2 \pi} d \phi \int_{-\frac{L}{2}}^{\frac{L}{2}} d z \frac{r(r \cos \phi-R)}{\left(R^{2}+r^{2}+z^{2}-2 R r \cos \phi\right)^{\frac{7}{2}}}
$$

where $M_{e}$ is the mass of the electron, $f_{e}^{(\text {sample) }}$ and $f_{e}^{(\mathrm{cyl})}$ are respectively the electronic mass fraction of the sample and the one of the cylinder, $\rho^{(\mathrm{cyl})}$ is the mass density of the cylinder, $R_{0}$ is its interior radius, $d$ its wall thickness and $L$ its length, and $R$ is the distance between the sample and the interior cylinder wall. Feeding the actual values for these parameters and doing the integral, one can check that the experimental results are compatible with an $\alpha_{g}<1.27$. This bound is very poor. Although it is many orders of magnitude better than the astronomical bounds discussed before, again the conclusion is that these forces are macroscopically very efficiently shielded, so that only detailed microscopic physics can provide useful information about them.

Finally, we would like to compare the effect of pseudoscalar forces with the well known Casimir effect [9]. This very tiny effect, due to quantum fluctuations of the electromagnetic field, has been experimentally verified [10] and we wish to quantify the strength of our putative force compared to the Casimir force. 
Consider the force between two large parallel plates of thickness $d$ separated by a distance $a$. Ignoring edge effects (i.e. a,d much smaller than the size of the plates) one obtains for copper,

$$
\left(\frac{F}{S}\right)_{C u}=-1.4 \times 10^{-3} \alpha_{g}^{2}\left(\frac{1}{(a+2 d)^{2}}+\frac{1}{a^{2}}-\frac{2}{(a+d)^{2}}\right) \quad N / m^{2}
$$

for the attractive force per unit surface area where $a, d$ are in $\mu m$. Of course, we took copper just to be definite but it could equally well be any other material, conducting or dielectric. Let us compare this to the Casimir force between the plates (in this case the plates must be necessarily conducting)

$$
\frac{F_{C a s}}{S}=-1.3 \times 10^{-3} \frac{1}{a^{4}} \mathrm{~N} / \mathrm{m}^{2}
$$

Notice that for very thin plates $(d \ll a)$ the $a$ behaviour of both forces is identical.

These results imply that a verification of the Casimir effect with, say, a $10 \%$ precision, demands $\alpha_{g}<0.27$, i.e. less than 1 , but still a poor bound.

We note in passing that since $\alpha=\frac{1}{137}$ is always less than the bounds for $\alpha_{g}$ obtained, the equivalent electron Pauli magnetic moment effect is also undetectable.

To summarize, we have shown that residual long-range attractive and composition dependent forces between macroscopic unpolarised neutral bodies which are associated to spin dependent couplings of fermions to massless bosons -a quantum mechanical effect due to the exchange of two quanta "at a time" - are very efficiently shielded over an enormous range of distances: from astronomical scales down to the micron scale.

Aknowledgement

This work has been partially supported by CICYT under project number AEN-93-0474.

\section{References}

[1] For a review, see

E. Fischbach and C. Talmadge, Nature $\underline{356}$ (1992) 207.

E. Fischbach, G.T. Gillies, D.E. Krause, J.G. Schwan and C. Talmadge, Metrologia $\underline{29}$ (1992) 213.

[2] J.Frieman and B. Gradwohl, Phys. Rev. Lett. $\underline{67}$ (1991) 2926.

J. Frieman and B. Gradwohl, Ap. J. $\underline{398}$ (1992) 407.

C.W. Stubbs, Phys. Rev. Lett. $\underline{70}$ (1993) 119.

G. Smith, E.G. Adelberger, B.R. Heckel and Y. Su, Phys. Rev. Lett. 70 (1993) 123.

[3] T.D. Lee and C.N. Yang, Phys. Rev. $\underline{98}$ (1955) 50. 
[4] A.D. Dolgov and Ya.B. Zeldovich, Rev. Mod. Phys. $\underline{53}$ (1981) 1.

L.B. Okun, Yad. Fiz. 10 (1969) 358. [Sov. J. Nucl. Phys. $\underline{3}$ (1966) 837].

J.E. Moody and F. Wilczek, Phys. Rev. D30 (1984) 130.

A. de Rújula, Phys. Lett. 180B (1986) 213.

C. Talmadge, J.P. Berthias, R.W. Hellings and E.M. Standish, Phys. Rev. Lett. $\underline{61}$ (1988) 1159.

[5] For a review, see G.G. Raffelt, Phys. Rep. 198 (1990) 1.

[6] J. J. Sakurai, Advanced Quantum Mechanics, Addison-Wesley (1967).

[7] G. Feinberg and J. Sucher, Phys. Rev. D45 (1992) 2493.

G. Feinberg, J. Sucher and C.K. Au, Phys. Rep. 180 (1989) 83.

[8] J.S. Hoskins, R.D. Newman, R. Spero and J. Schultz, Phys. Rev. D32 (1985) 3084.

Y.T. Chen, A.H. Cook, A.J.F. Metherell, Proc. R. Soc. A394 (1984) 47.

[9] H. B. G. Casimir, Proc. Kon. Ned. Akad. Wet. $\underline{51}$ (1948) 793.

[10] M.J. Sparnaay, Physica 24 (1958) 751. 